\title{
Visualization of bond rearrangements in acetylene using near single-cycle laser pulses
}

\author{
Christian Burger, ${ }^{\text {aab }}$ Nora G. Kling, ${ }^{a}$ Robert Siemering, ${ }^{c}$ Ali S. Alnaser, ${ }^{\text {bd }}$ \\ Boris Bergues, ${ }^{b}$ Abdallah M. Azzeer, ${ }^{e}$ Robert Moshammer, ${ }^{f}$ Regina de \\ Vivie-Riedle, ${ }^{c}$ Matthias Kübel ${ }^{\mathrm{a}}$ and Matthias F. Kling ${ }^{\mathrm{ab}}$
}

Received 15th April 2016, Accepted 10th May 2016

DOI: $10.1039 / \mathrm{c} 6 \mathrm{fd} 00082 \mathrm{~g}$

The migration of hydrogen atoms resulting in the isomerization of hydrocarbons is an important process which can occur on ultrafast timescales. Here, we visualize the lightinduced hydrogen migration of acetylene to vinylidene in an ionic state using two synchronized 4 fs intense laser pulses. The first pulse induces hydrogen migration, and the second is used for monitoring transient structural changes via Coulomb explosion imaging. Varying the time delay between the pulses reveals the migration dynamics with a time constant of $54 \pm 4$ fs as observed in the $\mathrm{H}^{+}+\mathrm{H}^{+}+\mathrm{CC}^{+}$channel. Due to the high temporal resolution, vibrational wave-packet motions along the $\mathrm{CC}$ - and $\mathrm{CH}$ bonds are observed. Even though a maximum in isomerization yield for kinetic energy releases above $16 \mathrm{eV}$ is measured, we find no indication for a backwards isomerization - in contrast to previous measurements. Here, we propose an alternative explanation for the maximum in isomerization yield, namely the surpassing of the transition state to the vinylidene configuration within the excited dication state.

\section{Introduction}

The photo-excitation of hydrocarbons and the reactions of their nuclei have been studied extensively in order to reveal the underlying physical processes, such as vibrations, ${ }^{1,2}$ rotations, ${ }^{3}$ bond rearrangements ${ }^{4}$ and molecular fragmentation. ${ }^{5}$ Each process has a corresponding natural timescale, with the fastest nuclear motions occurring in less than $10 \mathrm{fs}$, and therefore ultrafast laser pulses are ideally suited for their study. Furthermore, bond rearrangement and molecular

\footnotetext{
${ }^{a}$ Department of Physics, LMU Munich, D-85748 Garching, Germany. E-mail: christian.burger@mpq.mpg.de ${ }^{b}$ Max Planck Institute of Quantum Optics, D-85748 Garching, Germany 'Department of Chemistry and Biochemistry, LMU Munich, D-81377 Munich, Germany ${ }^{d}$ Physics Department, American University of Sharjah, POB2666, Sharjah, UAE ${ }^{e}$ Department of Physics and Astronomy, King-Saud University, Riyadh 11451, Saudi Arabia ${ }^{f}$ Max Planck Institute of Nuclear Physics, D-69117 Heidelberg, Germany
} 
fragmentation of hydrocarbons can be influenced by tuning the laser parameters. For example, Xie et al. showed that they can control the fragmentation pathways in ethylene by varying the laser pulse duration, ${ }^{6}$ and Alnaser et al. showed that they could steer the directional emission of the deprotonation from acetylene with the carrier-envelope phase (CEP) of few-cycle laser pulses. ${ }^{7}$ The second example was interpreted with a vibrational state coupling model. Recently, CEPcontrol has been extended to the isomerization of acetylene and allene, demonstrating the general importance of the vibrational state coupling mechanism. ${ }^{8}$

In fact, the process of hydrogen migration in acetylene has been the subject of numerous past studies, both theoretically ${ }^{9}$ and experimentally., ${ }^{2,10,11}$ More recent experiments have taken advantage of the Coulomb explosion imaging technique using a reaction microscope (REMI) in order to visualize the temporal evolution of the isomerization process in "real time". ${ }^{12}$ Specifically, the isomerization of acetylene into vinylidene has been visualized for the dissociation channel: $\mathrm{C}_{2} \mathrm{H}_{2}{ }^{3+}$ $\rightarrow \mathrm{H}^{+}+\mathrm{C}^{+}+\mathrm{CH}^{+} \cdot{ }^{12-15}$ The isomerization time was consistently found to be around $50 \mathrm{fs}$, independent from the chosen excitation wavelength and intensity, as well as independent of whether the dynamics occurred on the cation or dication intermediate states.

Here, we show how to exploit the additional channel $\mathrm{C}_{2} \mathrm{H}_{2}{ }^{3+} \rightarrow \mathrm{H}^{+}+\mathrm{H}^{+}+\mathrm{CC}^{+}$ to obtain a more complete understanding of the proton migration as suggested in ref. 16. We use ultrashort laser pulses as pump and probe pulses to visualize the migration process. After a short introduction into our experimental and theoretical methods we present our results on the triple coincidence channels, focusing on the comparison between the channels $\mathrm{H}^{+}+\mathrm{C}^{+}+\mathrm{CH}^{+}$and $\mathrm{H}^{+}+\mathrm{H}^{+}+$ $\mathrm{CC}^{+}$. Additionally, we determine the isomerization time for the latter channel, and by means of Newton diagrams we present snapshots of the hydrogen migration for various time delays between the pump and probe pulses.

\section{Experimental and theoretical methods}

\subsection{Experimental methods}

In order to investigate the hydrogen migration from acetylene to vinylidene, the experimental setup depicted in Fig. 1 was used. The titanium:sapphire system ("FEMTOPOWERTM HR $10 \mathrm{kHz}$ CEP4", Spectra-Physics) produces laser pulses at a $10 \mathrm{kHz}$ repetition rate with a pulse duration of $22 \mathrm{fs}$, a pulse energy of $800 \mu \mathrm{J}$ and a central wavelength of $790 \mathrm{~nm}$. The pulses are spectrally broadened by a $1 \mathrm{~m}$ long hollow-core-fiber with a $275 \mu \mathrm{m}$ inner core diameter, filled with $500 \mathrm{mbar}$ of argon with the final spectrum covering a range from $400 \mathrm{~nm}$ to $1000 \mathrm{~nm}$ with a new central wavelength of $690 \mathrm{~nm}$. Temporal compression is attained in a doubleangle chirped mirror compressor, ${ }^{17}$ while the fine tuning to 4 fs full-width halfmaximum (FWHM) is done with fused silica wedges. A variable neutral density filter allows us to change the intensity without changing the beam diameter or the dispersion and thus preserves the pulse parameters. The pulse duration is determined by transient-grating frequency-resolved optical gating (TG-FROG). ${ }^{18}$ The carrier-envelope-phase (CEP) is recorded by an $\mathrm{f}-2 \mathrm{f}$ interferometer throughout the measurement. ${ }^{19}$ The laser is split into two identical pump- and probe-pulses by a 50:50 Mach-Zehnder interferometer prior to the entrance window of a REMI (base pressure: $4 \times 10^{-10} \mathrm{mbar}$ ). The pulses are back-focused using a $12.5 \mathrm{~cm}$ focal length mirror onto a cold acetylene jet. The thickness of the 


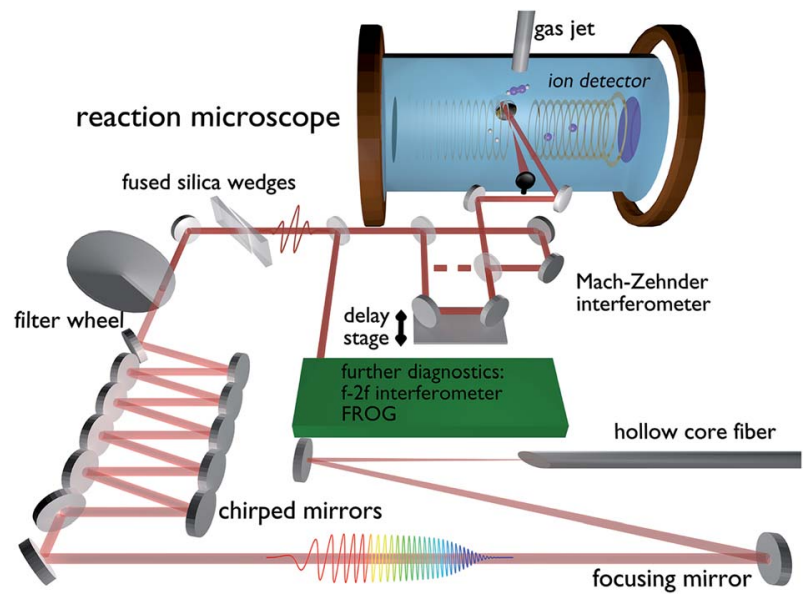

Fig. 1 Experimental setup including all major optical components after the amplifier and a schematic drawing of the experimental chamber.

jet can be varied using automated skimmers to maintain a constant overall count rate. Each pulse has an intensity around $5 \times 10^{14} \mathrm{~W} \mathrm{~cm}^{-2}$. The time delay between the pulses is continuously swept from -267 fs to $267 \mathrm{fs}$ in steps of 33 as. One period takes around 5 minutes, while the phase of both pulses changes by $2 \pi$ within $7 \mathrm{~s}$. This difference in timing ensures that no artificial CEP dependencies on the delay measurement are introduced, which is further verified by a flat CEP distribution for the complete delay range.

The data acquisition for the results shown here was carried out over three days and consists of four individual measurements, in between which the pulse duration and spatial overlap were verified to ensure similar parameters throughout the entire duration of the experiment.

In Table 1, the accumulated amount of events is shown for each run and each coincidence channel. The statistics indicate that the $\mathrm{H}^{+}+\mathrm{C}^{+}+\mathrm{CH}^{+}$channel has around $80 \%$ more counts than the $\mathrm{H}^{+}+\mathrm{H}^{+}+\mathrm{CC}^{+}$channel. The total statistics are about 5 times higher than previously reported, ${ }^{14}$ attributed to the high repetition rate laser and the long-term stability of the overall system. Such high statistics, combined with the high temporal resolution, allows us to investigate the vibrational motion within the intermediate state in addition to the isomerization process.

To combine the CEP-dependent data from each measurement, the relative phase for each scan is shifted to match an independent reference data set. This

Table 1 Presented data are combined from the following measurements

Coincidences per channel

\begin{tabular}{lllll} 
Measurement run & 1 & 2 & 3 & 4 \\
\hline $\mathrm{H}^{+}+\mathrm{C}^{+}+\mathrm{CH}^{+}$ & 245552 & 89274 & 32796 & 146586 \\
$\mathrm{H}^{+}+\mathrm{H}^{+}+\mathrm{CC}^{+}$ & 129131 & 55396 & 18770 & 80105 \\
$\mathrm{H}^{+}+\mathrm{H}^{+}+\mathrm{C}^{+}+\mathrm{C}^{+}$ & 53695 & 8156 & 5944 & 44955
\end{tabular}


reference is given by the strongest phase dependent signal, the double ionization asymmetry of acetylene, at a specific time delay (100 $\pm 10 \mathrm{fs})-$ far from the temporal overlap to avoid interference effects. The asymmetry is calculated by $A=\frac{N_{\text {left }}-N_{\text {right }}}{N_{\text {left }}+N_{\text {right }}}$, where $N_{\text {left }}$ and $N_{\text {right }}$ are the photo-ion yields to the left and right directions, respectively, along the polarization axis in the time-of-flight (TOF) spectrum. For further information see ref. 20. Furthermore, we checked that the measured data are independent of the sign of the delay. With this condition fulfilled, the data from negative and positive delays are combined to achieve better statistics.

Various dissociation channels from strong field pump-probe measurements can be identified in the coincidence spectra. Of these, the channels involving a clear isomerization component are:

$$
\begin{gathered}
\mathrm{C}_{2} \mathrm{H}_{2}^{2+} \rightarrow \mathrm{C}^{+}+\mathrm{CH}_{2}^{+} \\
\mathrm{C}_{2} \mathrm{H}_{2}^{2+} \rightarrow \mathrm{C}_{2}^{+}+\mathrm{H}_{2}^{+} \\
\mathrm{C}_{2} \mathrm{H}_{2}^{3+} \rightarrow \mathrm{H}_{2}^{+}+\mathrm{C}^{+}+\mathrm{C}^{+} \\
\mathrm{C}_{2} \mathrm{H}_{2}^{3+} \rightarrow \mathrm{H}^{+}+\mathrm{C}^{+}+\mathrm{CH}^{+} \\
\mathrm{C}_{2} \mathrm{H}_{2}^{3+} \rightarrow \mathrm{H}^{+}+\mathrm{H}^{+}+\mathrm{CC}^{+} \\
\mathrm{C}_{2} \mathrm{H}_{2}{ }^{4+} \rightarrow \mathrm{H}^{+}+\mathrm{H}^{+}+\mathrm{C}^{+}+\mathrm{C}^{+}
\end{gathered}
$$

Channel (i) permits us to measure the fragmentation yield of vinylidene and therefore to determine the average isomerization time to $52 \pm 15 \mathrm{fs}$, as investigated by Jiang et al. $^{21}$ Channels (ii) and (iii) are relatively weak, most likely because they require both hydrogen migration and bond formation between the hydrogens. Despite being interesting channels, the low statistics prevented us from being able to determine much about them and therefore, they are left out of the main discussion here. Channels (iv) and (v) have excellent statistics, and while channel (iv) has been extensively studied (see ref. 12-15), the investigation of channel (v) has been proposed ${ }^{\mathbf{1 6}}$ but reports have been lacking so far. Here, we compare both channels and highlight the advantages of channel $\mathrm{H}^{+}+$ $\mathrm{H}^{+}+\mathrm{CC}^{+}$. For channel (vi), preliminary analysis also shows signs of isomerization. However, due to the additional complexity of the quadruply charged parent ion, and the data analysis of the four-body coincidence, it will not be discussed in detail here. Instead, our main focus is on channels (iv) and (v). These two channels enable us to compare our measurements with previous experiments on the $\mathrm{H}^{+}+\mathrm{C}^{+}+\mathrm{CH}^{+}$channel and gain further information by analyzing the $\mathrm{H}^{+}+\mathrm{H}^{+}+\mathrm{CC}^{+}$channel. However, we would also like to point out that a considerable advantage of channel (v) lies in the large difference in mass of the $\mathrm{C}_{2}^{+}$and $\mathrm{H}^{+}$fragments, avoiding any overlap of their TOF's. This is especially important in contrast to the $\mathrm{H}^{+}+\mathrm{C}^{+}+\mathrm{CH}^{+}$channel, where the $\mathrm{C}^{+}$and $\mathrm{CH}^{+}$fragment have overlapping TOF's, which make the molecular reconstruction more difficult. 


\subsection{Theoretical methods}

We used a Complete Active Space Self-Consistent Field model (CASSCF) to calculate the potential energy surfaces for the ground states of the neutral, cation, dication, and trication states, as well as for different excited states. The active space provided for the electron correlation was adapted to the number of the valence electrons and consists of the same 10 molecular orbitals and all electrons except the 1s-core electrons of the carbon atoms. The calculations were performed using the program package MOLPRO with a $6-311++\mathrm{G}^{* *}$ basis set. ${ }^{22}$ Using this model we utilize the precise description of excited states to calculate the molecular configurations. These results enable us to understand the pathway of the migrating hydrogen atom within the dication state.

\section{Results and discussion}

In Fig. 2, the kinetic energy release (KER) of the three-fold coincidence channels is shown as a function of the time delay between the pump and probe pulses. The color scale in (a) and (b) indicates the yield, which is normalized to one for each time step. The integrated counts for each time step without normalization are plotted below in (c) and (d). Clearly visible in Fig. 2(a) and (b) is the strong signal at 16-17 eV that is largely delay independent and the contributions at lower KER values which are time dependent. Both areas can be separated at a KER of $13 \mathrm{eV}$, indicated by the black dashed line, which is later used for energy filtering. The low KER features are the result of bond stretching induced by the pump pulse, as seen in many other pump-probe studies on dissociating molecules. ${ }^{23-25}$

While the $\mathrm{H}^{+}+\mathrm{C}^{+}+\mathrm{CH}^{+}$channel is sensitive to both the $\mathrm{CC}$ - and $\mathrm{CH}$-bonds, the $\mathrm{H}^{+}+\mathrm{H}^{+}+\mathrm{CC}^{+}$channel exclusively probes the $\mathrm{CH}$-bonds. The slower temporal evolution of the low KER contribution in the $\mathrm{H}^{+}+\mathrm{C}^{+}+\mathrm{CH}^{+}$channel indicates a slower bond stretching of the CC-bond in comparison to the $\mathrm{CH}$-bond, as expected. Furthermore, the data shown in Fig. 2 contains two independent indications for a wave-packet motion in the intermediate state. First, we present in Fig. 2(c) and (d) the yield as a function of the pulse delay which shows
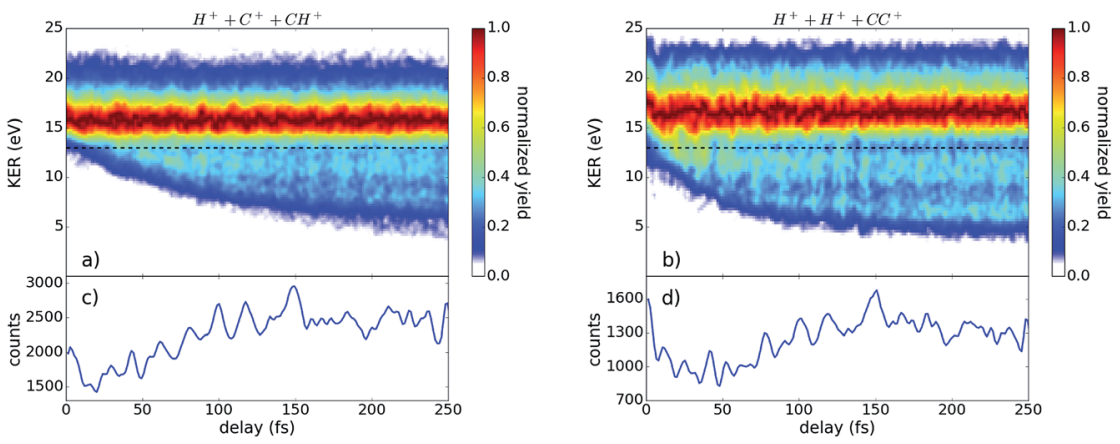

Fig. 2 The yield of (a) $\mathrm{H}^{+}+\mathrm{C}^{+}+\mathrm{CH}^{+}$and (b) $\mathrm{H}^{+}+\mathrm{H}^{+}+\mathrm{CC}^{+}$as a function of the kinetic energy release (KER) and pulse delay. The yield for each time bin is normalized to its peak, revealing a small oscillation of the KER with the delay. The black dashed line indicates the separation of the regions investigated in Fig. 4. The total yield corresponding to (a) and (b) is plotted in (c) and (d), respectively. 
a fine-structured oscillation. Secondly, we observe a periodical oscillation of the maximum position of the KER distribution around $17 \mathrm{eV}$ which becomes observable only by using ultrashort pulses. ${ }^{26,27}$ Applying a Fourier analysis to the oscillations of the highest KER values, as well as to the oscillations of the total yield dependent of the pulse delay, reveals three major contributions: $11.5 \mathrm{fs} \pm$ $1.7 \mathrm{fs}, 20.4 \mathrm{fs} \pm 1.5 \mathrm{fs}$, and $33.4 \mathrm{fs} \pm 1.2 \mathrm{fs}$. To attribute these experimentally determined periods to the corresponding wave-packet motion we calculated the periods of various modes within the neutral, cation, and dication, presented in Table 2 .

Comparing our experimental results to the calculated modes in Table 2 we identify the $11.5 \mathrm{fs} \pm 1.7$ fs oscillation with a $\mathrm{CH}$-bond stretching mode and the $20.4 \mathrm{fs} \pm 1.5 \mathrm{fs}$ vibration with a CC-bond stretching mode within the dication. Thus we can directly observe the $\mathrm{CH}$ - and CC-vibrations in acetylene via strongfield pump-probe measurements of the fragment ions. The slowest experimentally observed oscillation of $33.4 \mathrm{fs} \pm 1.2 \mathrm{fs}$ is not identified by our theoretical calculations as the various bending modes are slower than $40 \mathrm{fs}$, while the stretching modes are too fast to explain our results. Even though we cannot link the observed oscillation to one distinct mode, it is likely that we observe a combination of the stretching modes resulting in slower oscillations. To investigate the populated state further, we present in Fig. 3 the different potential energy curves of acetylene.

Selected potential energy curves along the $\mathrm{CH}$-bond internuclear distance are depicted in Fig. 3 along with a sketch of the various configurations of acetylene in the intermediate state. First, the pump pulse is ionizing the molecule, bringing it into an intermediate state, either the cation or dication state, where hydrogen migration can take place. The probe pulse ionizes the molecule further until it dissociates from a triply or even higher charged state. Depending on the excitation wavelength and intensity, different populated intermediate states have been reported so far. While using XUV or UV light for excitation results in a populated cation state, ${ }^{14,15,28}$ IR strong field excitation populates the dication state. ${ }^{12,13}$ In our experimental setup, a dication state is the most probable to be populated, as we use identical pump and probe pulses (see Fig. 3). Reachable are the ground state $\mathrm{X}^{3} \Sigma_{\mathrm{g}}{ }^{-}$and excited states $\mathrm{X}^{1} \Delta_{\mathrm{g}}$, and $\mathrm{A}^{3} \Pi_{\mathrm{u}}$. The latter is purely repulsive along the $\mathrm{C}-\mathrm{H}$ bond.

Pumping the molecule into an excited dication state can trigger several motions such as vibration, dissociation, rotation and isomerization, as depicted

Table 2 Calculated frequencies of different modes in the neutral, cation, and dication

\begin{tabular}{llll}
\hline & \multicolumn{2}{c}{ Period of oscillation (fs) } \\
\cline { 2 - 4 } Mode & Neutral & Cation & Dication \\
\hline Trans-bending $(x-z$ and $y-z)$ & $59.2 \pm 0.6$ & $40.4 \pm 0.5$ and $70.1 \pm 0.7$ & $52.2 \pm 0.6$ \\
Cis-bending $(x-z$ and $y-z)$ & $48.1 \pm 0.5$ & $40.5 \pm 0.5$ and $45.4 \pm 0.5$ & $47.0 \pm 0.5$ \\
CC-stretching & $16.3 \pm 0.5$ & $23.0 \pm 0.5$ & $21.6 \pm 0.5$ \\
Antisymmetric CH- & $10.0 \pm 0.4$ & $11.2 \pm 0.4$ & $11.4 \pm 0.4$ \\
stretching & & & $11.0 \pm 0.4$ \\
Symmetric CH-stretching & $9.7 \pm 0.4$ & $10.9 \pm 0.4$ &
\end{tabular}




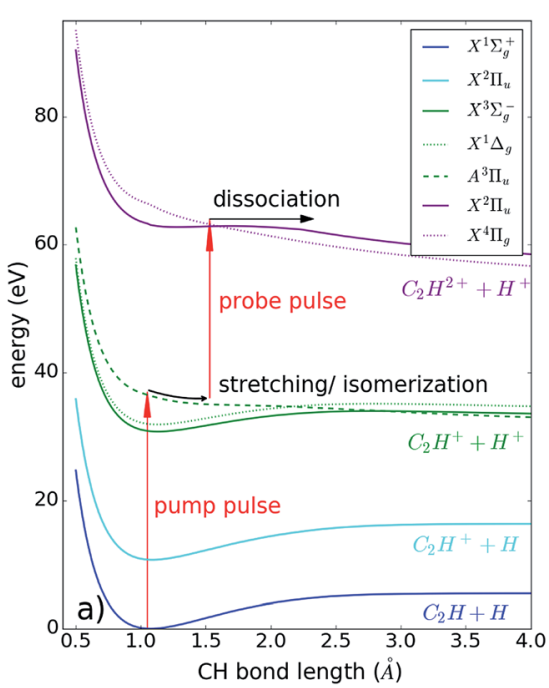

Fig. 3 (a) Potential energy curves for the neutral (blue), cation (cyan), dication (green), and trication (purple) ground states and select excited states as a function of the internuclear distance of one $\mathrm{CH}$-bond. (b) Sketch of the molecular configurations at different stages of the experiment. A pump pulse doubly ionizes the molecule and excites it to a reactive state that permits hydrogen migration. The probe pulse ionizes the molecule further bringing it into a triply charged dissociative state. The angles $\alpha$ and $\theta$ are used to distinguish between the acetylene and vinylidene configurations.

schematically in Fig. 3(b). Experimentally, we observe the 3D momenta of each ionic fragment, enabling us to reconstruct the molecular arrangement at the time of Coulomb explosion. Together with the KER, this information allows us to disentangle the various molecular motions.

To describe isomerization, it is useful to define the angles $\alpha$ and $\theta$ of the migrating hydrogen (see Fig. 3(b)). In order to compare our results to previous measurements, $\alpha$ is defined as in ref. 12 and 13 and describes the angle between the proton and the carbon ion with respect to the center of gravity of the molecule (see Fig. 3(b)). The angle $\theta$ corresponds to the angle in between both protons seen from the $\mathrm{C}_{2}{ }^{+}$ion. Using the $\mathrm{C}_{2}{ }^{+}$ion as a center enables us to directly compare our results with theoretical calculations performed on the dication of acetylene. Both angles are calculated via: $\alpha, \theta=\cos ^{-1}\left(\frac{\vec{p}_{1} \cdot \vec{p}_{2}}{\left|\vec{p}_{1}\right|\left|\vec{p}_{2}\right|}\right)$, where $\vec{p}_{1}$ and $\vec{p}_{2}$ are the momenta of the hydrogen and carbon ions for channel (iv) and of both protons for channel (v).

By this definition of angles, we assign $\alpha$ at around $0^{\circ}$ and $\theta$ at $180^{\circ}$ to the acetylene configuration and $\alpha$ above $90^{\circ}$ and $\theta$ below $90^{\circ}$ to vinylidene. In the following part we will extend our analysis to the reconstruction of the molecular configuration - the angles $\alpha$ and $\theta$ provide us with the information about the angular position of the migrating proton.

In Fig. 4, the fragmentation yield as a function of the angles $\alpha$ and $\theta$ and the pulse delay is shown for both coincidence channels with two different energy filters. The energy filter separates the time independent KER region (above $13 \mathrm{eV}$ ) 

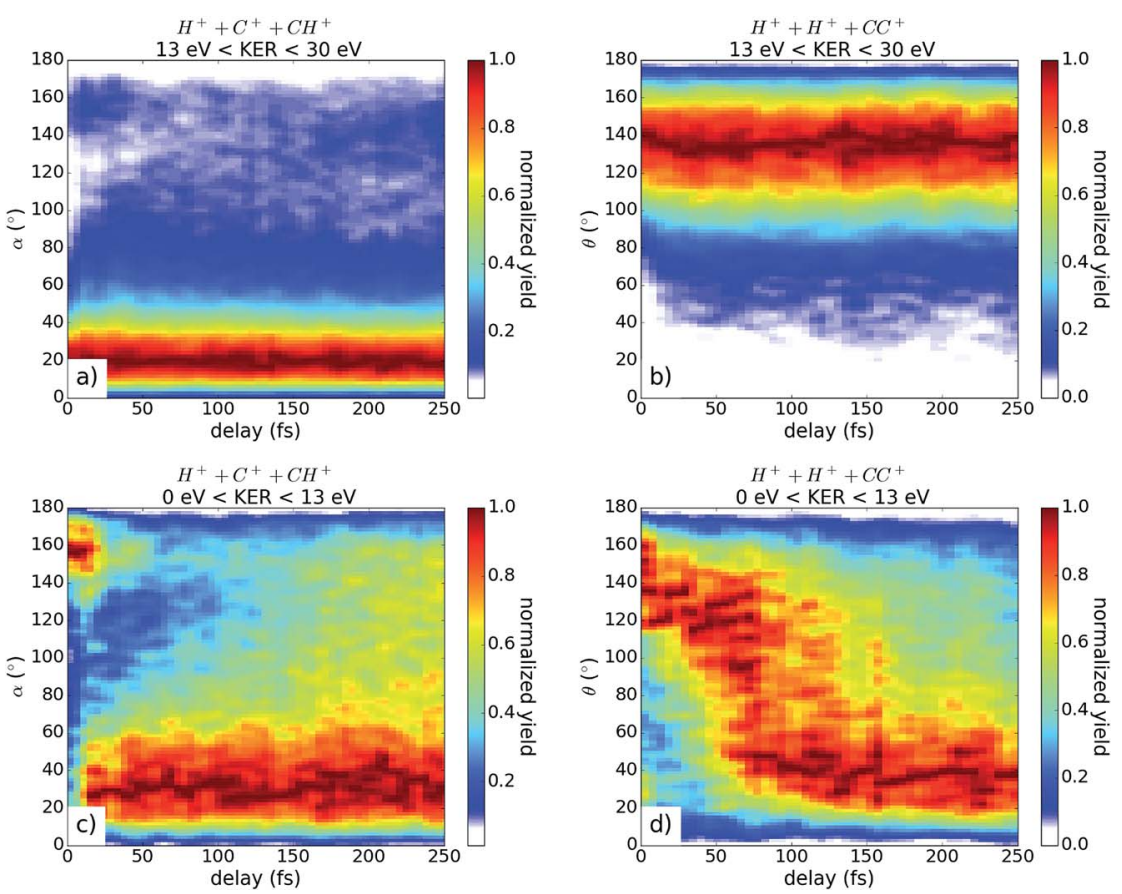

Fig. 4 The fragmentation yield is shown as a function of pump-probe delay and the angle $\alpha$ for the channel $\mathrm{H}^{+}+\mathrm{C}^{+}+\mathrm{CH}^{+}$in panels (a) and (c), and $\theta$ for channel $\mathrm{H}^{+}+\mathrm{H}^{+}+\mathrm{CC}^{+}$in panels (b) and (d). The applied KER filters (indicated above each plot) are used to separate the bound (13-30 eV, (a) and (b)) from the dissociating contribution (0-13 eV, (c) and (d)). The contributions in (a) and (c) at large angles and small delays are attributed to an artifact (see text).

from the dissociating contributions at lower KER values (see Fig. 2). For the high KER region in both channels, the angles stay relatively constant with broad angular distributions around $20^{\circ}$ and $140^{\circ}$, respectively, indicating that the molecules tend to stay in the acetylene configuration. These results fit very well to the calculated configuration of excited acetylene molecules within the dication, where the hydrogen atoms are bent with respect to the CC axis. Likely, the excitation energy is thereby stored in a wave-packet motion in the acetylene configuration, which results in a widespread momenta distribution around the acetylene configuration, visible here by the broad angular distribution.

For the low KER contributions plotted in Fig. 4(c) and (d), a clear time dependence is observed showing the movement to smaller angles. This indicates the isomerization to the vinylidene configuration. In contrast to the $\mathrm{H}^{+}+\mathrm{C}^{+}+\mathrm{CH}^{+}$ channel, the majority of the molecules change their molecular configuration in the $\mathrm{H}^{+}+\mathrm{H}^{+}+\mathrm{CC}^{+}$channel (see Fig. 4(d)). After approximately $100 \mathrm{fs}$, more than $60 \%$ of all molecules in the $\mathrm{H}^{+}+\mathrm{C}^{+}+\mathrm{CH}^{+}$channel have reached the vinylidene configuration. We note that in contrast to ref. 12, the migration back to the acetylene configuration is not observed, but rather, the molecules tend to favor the vinylidene configuration for the remaining duration of the scanned time delay. We note that this discrepancy could, at least in part, be due to the fact that 
Hishikawa et al. used a background subtraction, which accounted for three-fold coincidences from only one pulse. ${ }^{12}$

For the coincidence channel $\mathrm{H}^{+}+\mathrm{C}^{+}+\mathrm{CH}^{+}$, it is visible that we have a considerable amount of molecules in the vinylidene configuration at small time delays. This contribution results from the overlap in the TOF spectrum for the slow $\mathrm{C}^{+}$fragments and the fast $\mathrm{CH}^{+}$fragments. Inevitably, the identification of the two fragments in the data analysis is convoluted, potentially leading to a wrong assessment of the molecular configuration. For the $\mathrm{H}^{+}+\mathrm{H}^{+}+\mathrm{CC}^{+}$channel, the TOF's of each fragment are clearly separated, and the assessment of the molecular configuration is thus much more reliable.

Using the angular information as a filter, the molecules which ultimately dissociated from the vinylidene configuration are separated from those dissociated from the acetylene configuration. For channel $\mathrm{H}^{+}+\mathrm{C}^{+}+\mathrm{CH}^{+}$, all angles $\alpha$ above $90^{\circ}$ (see Fig. 3(b)) are referred to as vinylidene while all angles below $90^{\circ}$ are attributed to the acetylene configuration. For channel $\mathrm{H}^{+}+\mathrm{H}^{+}+\mathrm{CC}^{+}$, the vinylidene configuration is reached for all angles $\theta$ below $90^{\circ}$ (see Fig. 3(b)) and all angles above $90^{\circ}$ correspond to the acetylene configuration. The resulting KER distribution is plotted versus the pulse delay in Fig. 5 .

In Fig. 5, the yield is plotted as a function of the KER and time delay for molecules in the vinylidene configuration. Compared to Fig. 2, the contribution at 16-17 eV is considerably weaker, while the delay dependent contribution below 16 eV becomes clearly visible. For the investigated pump-probe delays, this indicates that the isomerized molecules in the dication dissociate too fast to allow for a back migration of the proton into an acetylene-like configuration.

Taking a closer look at Fig. 5(a), one can see that the KER extends to higher values at delays between 0 fs and $100 \mathrm{fs}$, marked by an arrow in the plot. This increase in KER values in channel $\mathrm{H}^{+}+\mathrm{C}^{+}+\mathrm{CH}^{+}$, as well as the delay dependence in both KER plots in Fig. 5 , can be well explained within the $\mathrm{A}^{3} \Pi_{\mathrm{u}}$ excited dication state, where an isomerization can take place as shown by theoretical calculations, see Fig. $6 .{ }^{29}$

According to our calculations in full dimension, acetylene in the $\mathrm{A}^{3} \Pi_{\mathrm{u}}$ state relaxes into the two local minima $\mathrm{t}^{\prime}$ and $\mathrm{t}^{\prime \prime}$ with bent configurations having
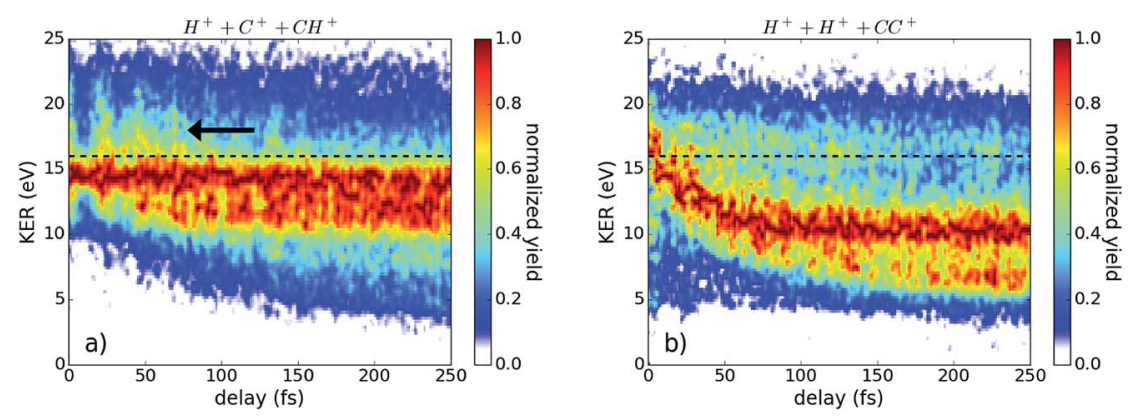

Fig. 5 Kinetic energy release from (a) $\mathrm{H}^{+}+\mathrm{C}^{+}+\mathrm{CH}^{+}$and (b) $\mathrm{H}^{+}+\mathrm{H}^{+}+\mathrm{CC}^{+}$versus the pulse delay, fulfilling the condition: $\alpha>90^{\circ}$ and $\theta<90^{\circ}$, indicating the molecule dissociated from the vinylidene configuration. The dashed black lines indicates the new energy filter used for Fig. 7, while the arrow points to an area of interest used to identify the intermediate state, as explained in the text. 


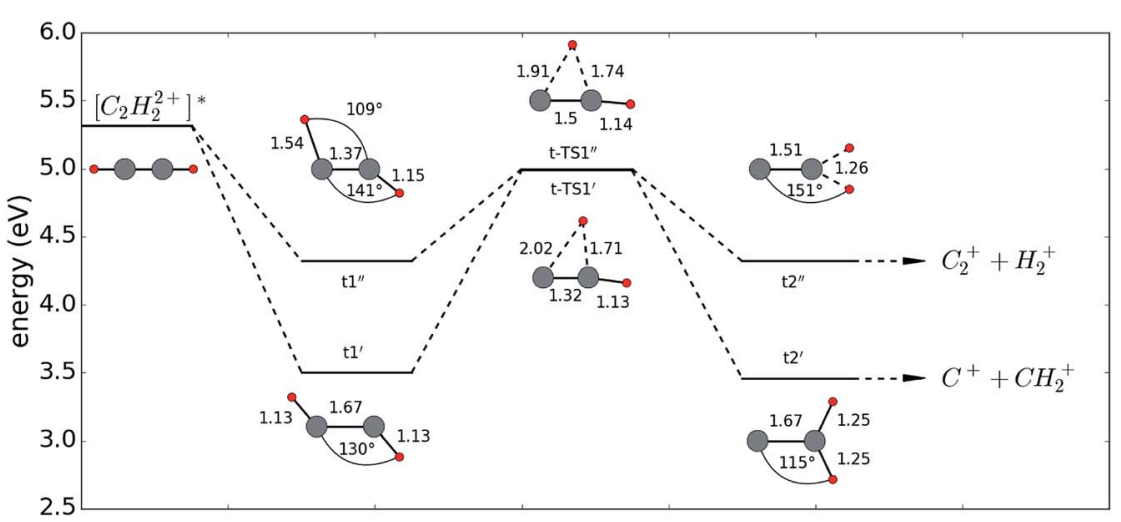

Fig. 6 Sketch of the energies and corresponding configurations of an acetylene molecule in the excited dication state. All configurations were calculated using the CASSCF $[8,10]$ model with a $6-311++G^{* *}$ basis set, where the bond lengths are given in units of $10^{-10} \mathrm{~m}$. The idea for this figure was taken from ref. 29.

angles around $140^{\circ}$ between the $\mathrm{CH}$-and CC-bonds. From these local minima, the energetically higher lying transition states t-TS1' and t-TS1" have to be overcome. The transition states are close in energy, the angle between both protons is around $90^{\circ}$, and the $\mathrm{CH}$-bond of the migrating proton is elongated while the CCbond is slightly shortened. In a last step, the molecule finishes its migration into vinylidene from where it can dissociate in either of two channels: $\left[\mathrm{C}_{2} \mathrm{H}_{2}{ }^{2+}\right]^{*} \rightarrow \mathrm{C}_{2}{ }^{+}$ $+\mathrm{H}_{2}{ }^{+}$or $\rightarrow \mathrm{C}^{+}+\mathrm{CH}_{2}{ }^{+}$. The probe pulse in our experiment is used to further ionize and dissociate the $\mathrm{H}_{2}{ }^{+}$or $\mathrm{CH}_{2}^{+}$, respectively. These dissociations are very well observed within both cases, visible in the time dependent low KER regions, whereas the intermediate transition states $\mathrm{t}-\mathrm{TS}^{\prime}{ }^{\prime}$ and $\mathrm{t}-\mathrm{TS} 1^{\prime \prime}$ are observed in the following two ways. Within the $\mathrm{H}^{+}+\mathrm{H}^{+}+\mathrm{CC}^{+}$channel, the stretched $\mathrm{CH}$-bond of the migrating proton is probed, resulting in the rapidly decreasing KER within the first 100 fs. In the $\mathrm{H}^{+}+\mathrm{C}^{+}+\mathrm{CH}^{+}$channel, the CC-bond can be probed resulting in a higher KER, seen at around 40 fs (see arrow in Fig. 5). This effect cannot be observed as easily as the $\mathrm{CH}$-bond stretching because within this channel both effects occur simultaneously, washing out the signal as one contribution delivers higher KER (short CC-bonds) while the other yields lower KERs (long CH-bonds).

Since we can follow the isomerization dynamics in our pump-probe experimental scheme, we can also extract the isomerization time by measuring the isomer yield as a function of pulse delay. The isomer yield is calculated as the ratio of vinylidene molecules $\left(\alpha>90^{\circ}, \theta<90^{\circ}\right)$ to all triple coincidences for each channel. In Fig. 7 , the isomerization yield dependence on the pulse delay is shown for both investigated channels.

We investigated two different scenarios in Fig. 7. In the first scenario, we consider all isomerization counts appearing in Fig. 5 and in the second, we only consider the number of isomerization events that occurred with a KER above 16 $\mathrm{eV}$, indicated by the dashed line in Fig. 5 . For the first scenario, we see an increase of the isomer yield with pulse delay. In the $\mathrm{H}^{+}+\mathrm{H}^{+}+\mathrm{CC}^{+}$channel an asymptotic behavior is observed for long delays indicating that the final isomerization yield is around $30 \%$. Note that these $30 \%$ result from the investigation of all measured 

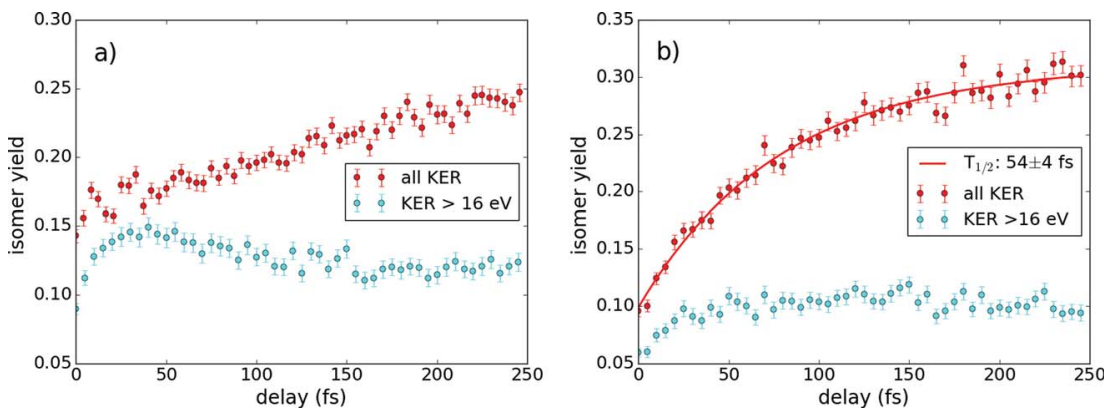

Fig. 7 Isomerization yield of (a) $\mathrm{H}^{+}+\mathrm{C}^{+}+\mathrm{CH}^{+}$and (b) $\mathrm{H}^{+}+\mathrm{H}^{+}+\mathrm{CC}^{+}$as a function of the pulse delay. The overall vinylidene yield divided by the total yield is shown in red, while an additional high-energy filter $(>16 \mathrm{eV})$ is applied to obtain the cyan colored data (error bars denote statistical errors). The solid red line indicates an exponential fit of the experimental data. Note that the isomer yield at zero pulse delay in (a) is overestimated due to the artifact discussed above.

data without applying any filter. Fitting this data with an exponential function allows us to determine the isomerization time to be $54 \pm 4 \mathrm{fs}$, which is consistent with previously reported isomerization times from both measurements and calculations. ${ }^{14,21,30,31}$

The $\mathrm{H}^{+}+\mathrm{C}^{+}+\mathrm{CH}^{+}$channel, in contrast, shows more of a linear trend for the pulse delays considered, which makes a determination of the isomerization time difficult. After $250 \mathrm{fs}$, the isomerization yield is at 25\%, which is considerably lower than for $\mathrm{H}^{+}+\mathrm{H}^{+}+\mathrm{CC}^{+}$. As the dynamics within the $\mathrm{H}^{+}+\mathrm{C}^{+}+\mathrm{CH}^{+}$channel seems to occur on longer timescales, it is expected that the isomerization yield would also saturate for longer pulse delays, from which we can only speculate that the isomerization time for this channel is longer than for the $\mathrm{H}^{+}+\mathrm{H}^{+}+\mathrm{CC}^{+}$channel.

For the second scenario, applying a high energy filter (KER $>16 \mathrm{eV}$ ), we reproduce previous measurements in the $\mathrm{H}^{+}+\mathrm{C}^{+}+\mathrm{CH}^{+}$channel showing a peak at $40 \mathrm{fs}$, which was interpreted as an isomerization maximum, where afterwards the proton may migrate back into the acetylene configuration. ${ }^{14}$ However, as we do not observe a similar behavior in the second coincidence channel, we propose an alternative explanation for the peak around $40 \mathrm{fs}$. In the intermediate state tTS1" (see Fig. 6), the CC-bond has a reduced equilibrium bond length, which may result in high KER values when dissociation is induced. For higher time delays the bond length increases again and the molecule stays within the vinylidene configuration without backwards migration. In other words, the peak of isomerization yield for the applied high KER filter is not due to a maximal isomerization yield, but rather to a CC vibrational motion. One would maybe think that a similar feature should appear in the $\mathrm{H}^{+}+\mathrm{H}^{+}+\mathrm{CC}^{+}$channel, but this channel is less sensitive to the CC-bond length, as this bond remains intact. Indeed, for the channel $\mathrm{H}^{+}+\mathrm{H}^{+}+\mathrm{CC}^{+}$, the isomerization yield fluctuates always around $10 \%$ without any other clear trend (see Fig. 7(b)).

Furthermore, we observe in Fig. 7 that the isomer yield at zero delay is always above $5 \%$ - independent of the investigated channel. This is likely due to a population of the trication where the isomerization takes place followed by Coulomb explosion. The direct population becomes possible by the high 

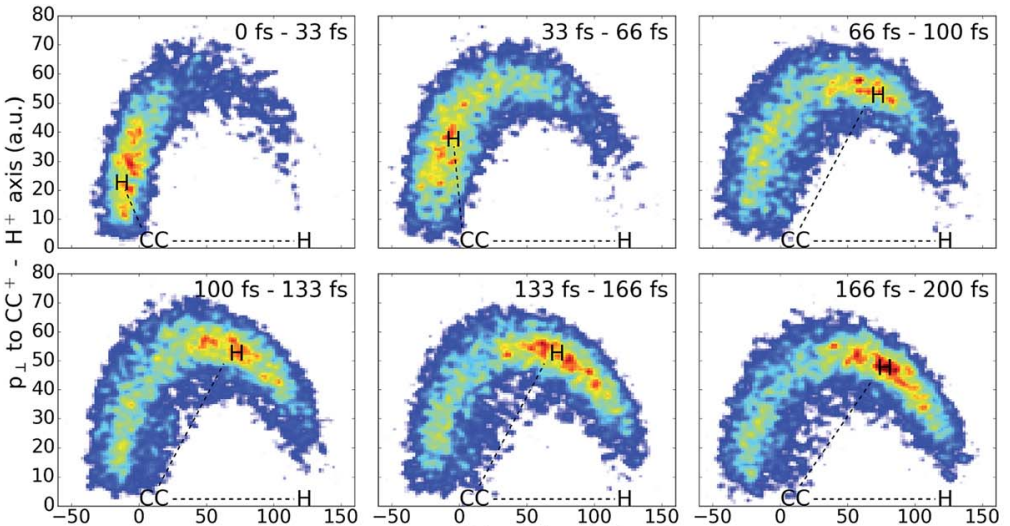

Paper
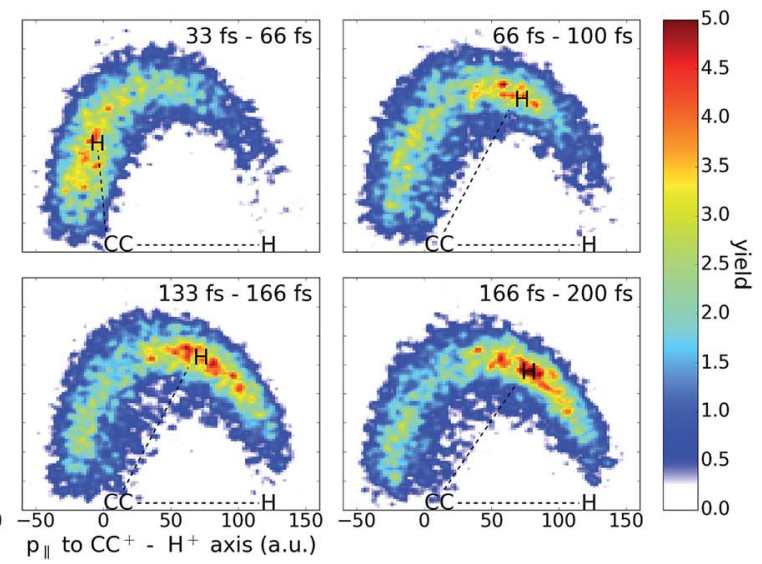

Fig. 8 Newton diagrams of the hydrogen migration for different pulse delays (indicated at the top right in each plot). As after 200 fs the Newton plots do not change anymore, only the first $200 \mathrm{fs}$ are presented here. The position of each atom is schematically shown as a guide to the eye.

intensities in the temporal overlap of both pulses. For higher pulse delays a direct ionization into the trication becomes unlikely as the intensity of one pulse alone is too low. The even higher isomer yields for Fig. 7(a) result from the artifact discussed above (see Fig. 4).

Finally, to visualize the hydrogen migration process, we plot a series of Newton diagrams for the $\mathrm{H}^{+}+\mathrm{H}^{+}+\mathrm{CC}^{+}$channel in Fig. 8. Filtering only the events of one dissociating contribution (with a KER between $8 \mathrm{eV}$ and $13 \mathrm{eV}$ ) highlights the isomerization process. Here, the Newton diagrams depict the 3D momenta in the following way: first, the momentum of the $\mathrm{CC}^{+}$ion is subtracted from the proton momenta in order to use the heaviest fragment as the center. Secondly, the momentum of the first proton is used as a reference to turn the coordinate system such that the $\mathrm{CC}^{+}-\mathrm{H}_{1}{ }^{+}$axis is fixed along the positive horizontal axis. Finally, the momentum components of the second hydrogen along this $\mathrm{CC}^{+}-\mathrm{H}_{1}{ }^{+}$axis $\left(p_{\|}\right)$ and the momentum perpendicular $\left(p_{\perp}\right)$ are plotted for different time delays. It is well visible that the isomerization takes place in the first $100 \mathrm{fs}$ until the majority of molecules are in the vinylidene configuration. For longer pulse delays, a maximal isomerization is reached and the momentum distribution stays mostly unchanged showing no sign of a backwards isomerization.

As mentioned in the Experimental methods section, the CEP was also recorded such that CEP effects can be investigated. We have not observed clear CEPdependent signatures in the pump-probe data, but it should be mentioned that the CEP effects in single pulse data were relatively small (asymmetries of around $7 \%$ have been detected). ${ }^{8}$ With the data at hand we cannot rule out that timedependent CEP-effects may be present, but if so, they are very small.

\section{Conclusion}

In summary, we have presented the molecular response in acetylene at various times after excitation by a near single-cycle pulse using the Coulomb explosion imaging technique. Investigating both major triple coincidence channels, we 
have observed the evolution of the hydrogen migration process for the isomerization to the vinylidene configuration. However, under our experimental conditions, no backwards isomerization to acetylene was observed. Despite this discrepancy, the isomerization time of the acetylene ion of $54 \pm 4 \mathrm{fs}$, which we have clearly determined via the $\mathrm{H}^{+}+\mathrm{H}^{+}+\mathrm{CC}^{+}$channel, is in excellent agreement with previous experimental studies ${ }^{21,30,31}$ and theoretical predictions. ${ }^{29}$ The maximum in isomerization yield is explained within the structural evolution of the excited dication, namely the surpassing of the energetically higher lying transition states t-TS1' and t-TS1" to the vinylidene configuration. The unprecedented temporal resolution in our experiment allowed us to identify vibrational wave-packet motions along the $\mathrm{CH}$ - and CC-bonds with periods of $11.5 \mathrm{fs} \pm 1.7 \mathrm{fs}$ and $20.4 \mathrm{fs} \pm 1.5 \mathrm{fs}$, respectively.

\section{Acknowledgements}

We are grateful to F. Krausz for his support and fruitful discussions. We are thankful for support by the Max Planck Society and by the DFG via LMUexcellent and via the Cluster of Excellence: Munich Center for Advanced Photonics (MAP). We are also grateful for support from the King-Saud University in the framework of the MPQ-KSU-LMU collaboration.

\section{References}

1 P. Jungner and L. Halonen, J. Chem. Phys., 1997, 55, 1680-1682.

2 R. Thissen, J. Delwiche, J. M. Robbe, D. Duflot, J. P. Flament and J. H. D. Eland, J. Chem. Phys., 1993, 99, 6590.

3 N. Ochi and S. Tsuchiya, Chem. Phys., 1991, 152, 319-336.

4 W. F. Manders and J. M. Bellama, Polymer, 1985, 23, 351-357.

5 C. Cornaggia, M. Schmidt and D. Normand, Phys. Rev. A, 1995, 51, 1431-1437.

6 X. Xie, S. Roither, M. Schöffler, E. Lötstedt, D. Kartashov, L. Zhang, G. G. Paulus, A. Iwasaki, A. Baltuška, K. Yamanouchi and M. Kitzler, Phys. Rev. $X, 2014,4,1-10$.

7 A. S. Alnaser, M. Kübel, R. Siemering, B. Bergues, N. G. Kling, K. J. Betsch, Y. Deng, J. Schmidt, Z. A. Alahmed, A. M. Azzeer, J. Ullrich, I. Ben-Itzhak, R. Moshammer, U. Kleineberg, F. Krausz, R. de Vivie-Riedle and M. F. Kling, Nat. Commun., 2014, 5, 3800.

8 M. Kübel, R. Siemering, C. Burger, N. G. Kling, H. Li, A. S. Alnaser, B. Bergues, S. Zherebtsov, A. M. Azzeer, I. Ben-Itzhak, R. Moshammer, R. de Vivie-Riedle and M. F. Kling, 2015, arxiv, 1508.04018.

9 D. Duflot, J.-M. Robbe and J.-P. Flament, J. Chem. Phys., 1995, 102, 355.

10 K. M. Ervin, J. Ho and W. C. Lineberger, J. Chem. Phys., 1989, 91, 5974.

11 M. P. Jacobson and R. W. Field, J. Phys. Chem. A, 2000, 104, 3073-3086.

12 A. Hishikawa, A. Matsuda, M. Fushitani and E. J. Takahashi, Phys. Rev. Lett., 2007, 99, 258302.

13 A. Hishikawa, A. Matsuda, E. J. Takahashi and M. Fushitani, J. Chem. Phys., 2008, 128, 084302.

14 H. Ibrahim, B. Wales, S. Beaulieu, B. E. Schmidt, N. Thiré, E. P. Fowe, É. Bisson, C. T. Hebeisen, V. Wanie, M. Giguére, J.-C. Kieffer, M. Spanner, A. D. Bandrauk, J. Sanderson, M. S. Schuurman and F. Légaré, Nat. Commun., 2014, 5, 4422. 
15 A. Matsuda, M. Fushitani, E. J. Takahashi and A. Hishikawa, Springer Proc. Phys., 2012, 125, 317-322.

16 A. S. Alnaser, I. Litvinyuk, T. Osipov, B. Ulrich, A. Landers, E. Wells, C. M. Maharjan, P. Ranitovic, I. Bochareva, D. Ray and C. L. Cocke, J. Phys. B: At., Mol. Opt. Phys., 2006, 39, 485-492.

17 V. Pervak, I. Ahmad, M. K. Trubetskov, a. V. Tikhonravov and F. Krausz, Opt. Express, 2009, 17, 7943-7951.

18 D. J. Kane and R. Trebino, IEEE J. Quantum Electron., 1993, 29, 571-579.

19 M. Schultze, A. Wirth, I. Grguras, M. Uiberacker, T. Uphues, A. Verhoef, J. Gagnon, M. Hofstetter, U. Kleineberg, E. Goulielmakis and F. Krausz, J. Electron Spectrosc. Relat. Phenom., 2011, 184, 68-77.

20 M. Kübel, K. J. Betsch, N. G. Johnson, U. Kleineberg, R. Moshammer, J. Ullrich, G. G. Paulus, M. F. Kling and B. Bergues, New J. Phys., 2012, 14, 093027.

21 Y. H. Jiang, A. Rudenko, O. Herrwerth, L. Foucar, M. Kurka, K. U. Kühnel, M. Lezius, M. F. Kling, J. Van Tilborg, A. Belkacem, K. Ueda, S. Düsterer, R. Treusch, C. D. Schröter, R. Moshammer and J. Ullrich, Phys. Rev. Lett., 2010, 105, 263002.

22 H.-J. Werner, P. J. Knowles, G. Knizia, F. R. Manby, M. Schütz, P. Celani, W. Györffy, D. Kats, T. Korona, R. Lindh, A. Mitrushenkov, G. Rauhut, K. R. Shamasundar, T. B. Adler, R. D. Amos, A. Bernhardsson, A. Berning, D. L. Cooper, M. J. O. Deegan, A. J. Dobbyn, F. Eckert, E. Goll, C. Hampel, A. Hesselmann, G. Hetzer, T. Hrenar, G. Jansen, C. Köppl, Y. Liu, A. W. Lloyd, R. A. Mata, A. J. May, S. J. McNicholas, W. Meyer, M. E. Mura, A. Nicklass, D. P. O'Neill, P. Palmieri, D. Peng, K. Pflüger, R. Pitzer, M. Reiher, T. Shiozaki, H. Stoll, A. J. Stone, R. Tarroni, T. Thorsteinsson and M. Wang, MOLPRO, Version 2006.1, a Package of Ab Initio Programs, 2006.

23 H. Stapelfeldt, E. Constant, H. Sakai and P. Corkum, Phys. Rev. A, 1998, 58, 426-433.

24 I. A. Bocharova, A. S. Alnaser, U. Thumm, T. Niederhausen, D. Ray, C. L. Cocke and I. V. Litvinyuk, Phys. Rev. A: At., Mol., Opt. Phys., 2011, 83, 1-17.

25 T. Ergler, A. Rudenko, B. Feuerstein, K. Zrost, C. D. Schröter, R. Moshammer and J. Ullrich, Phys. Rev. Lett., 2005, 95, 14-17.

26 M. Magrakvelidze, O. Herrwerth, Y. H. Jiang, A. Rudenko, M. Kurka, L. Foucar, K. U. Kühnel, M. Kübel, N. G. Johnson, C. D. Schröter, S. Düsterer, R. Treusch, M. Lezius, I. Ben-Itzhak, R. Moshammer, J. Ullrich, M. F. Kling and U. Thumm, Phys. Rev. A: At., Mol., Opt. Phys., 2012, 86, 1-11.

27 B. Fischer, M. Kremer, T. Pfeifer, B. Feuerstein, V. Sharma, U. Thumm, C. D. Schröter, R. Moshammer and J. Ullrich, Phys. Rev. Lett., 2010, 105, 1-4.

28 Y. H. Jiang, A. Senftleben, M. Kurka, A. Rudenko, L. Foucar, O. Herrwerth, M. F. Kling, M. Lezius, J. V. Tilborg, A. Belkacem, K. Ueda, D. Rolles, R. Treusch, Y. Z. Zhang, Y. F. Liu, C. D. Schröter, J. Ullrich and R. Moshammer, J. Phys. B: At., Mol. Opt. Phys., 2013, 46, 164027.

29 T. S. Zyubina, Y. A. Dyakov, S. H. Lin, A. D. Bandrauk and A. M. Mebel, J. Chem. Phys., 2005, 123, 134320.

30 Y. H. Jiang, A. Senftleben, A. Rudenko, M. E. Madjet, O. Vendrell, M. Kurka and K. Schnorr, 2010, arxiv, 1402.4874.

31 T. Osipov, C. L. Cocke, M. H. Prior, A. Landers, T. Weber, O. Jagutzki, L. Schmidt, H. Schmidt-Böcking and R. Dörner, Phys. Rev. Lett., 2003, 90, 233002. 